\title{
Un gène candidat pour la tumeur de Wilms
}

Le néphroblastome ou tumeur de Wilms (WT) est une tumeur rénale de type embyronnaire qui touche un enfant sur 10 000. Comme pour le rétinoblastome, il existe des formes sporadiques unilatérales et des formes héréditaires qui peuvent être bilatérales. Ces formes héréditaires sont pourtant beaucoup moins fréquentes que pour le rétinoblastome (30 à $40 \%)$, puisqu'environ $2 \%$ seulement des cas de WT correspondent à des cas familiaux et 1 à $2 \%$ des cas sont associés à une délétion constitutionnelle de la bande 11 p13. Cette délétion s'accompagne non seulement d'une prédisposition à la WT mais également d'une aniridie (absence totale ou partielle d'iris), d'anomalies génito-urinaires (GU) et d'une arriération mentale (syndrome WAGR). La région WAGR en 11 p13 contient donc un ensemble de gènes qui contribuent indépendamment au développement normal de l'iris, du rein, de l'appareil génito-urinaire et du système nerveux. Il n'est copendant pas impossible que la WT et les anomalies génito-urinaires reflètent l'effet pléiotrope d'une mutation touchant un seul et même gène [1].

Par analogie avec le modèle $\mathrm{d} u$ rétinoblastome (RB), il a été proposé que cette région $11 \mathrm{p} 13$ contienne un gène de type suppresseur de tumeur dont l'altération représenterait un événement clé dans le processus tumorigène. Dès 1972, Knudson et Strong proposaient, à juste titre, d'appliquer à la WT leur modèle des deux événements. Selon ce modèle, le premier événement est soit germinal, dans les cas héréditaires, soit somatique, dans les cas sporadiques ; le deuxième événement est nécessairement somatique. L'analyse du caryotype des tumeurs, par les techniques cytogénétiques, puis la mise

l'aide de marqueurs moléculaires, révélèrent effectivement l'existence d'anomalies chromosomiques spécifiques touchant le bras court du 11 . Pourtant il est apparu très vite que ces anomalies concernaient le plus souvent une région beaucoup plus distale située à quelques dizaines de mégabases, en 11p15.5. Sans toutefois remettre en cause le rôle du gène en $11 \mathrm{p} 13$, ces observations suggéraient la présence sur le bras court du 11 d'un deuxième gène impliqué dans la WT. L'existence de remaniements chromosomiques constitutionnels touchant cette même région chez des patients atteints de syndrome de Beckwith-Wiedemann (BWS) allait d'ailleurs dans le même sens. En effet ce syndrome polymalformatif présente lui aussi la particularité de prédisposer les enfants atteints à divers types de tumeurs dont la WT. Enfin, des études de familles avec prédisposition héréditaire à la tumeur de Wilms ont exclu la liaison avec des marqueurs de ces deux régions du bras court du 11, démontrant ainsi l'existence d'un troisième locus pour la prédisposition à la WT. Il n'en demeure pas moins que la région $11 \mathrm{p} 13$ abrite de toute évidence un gène impliqué dans la prédisposition à la WT. Ce gène serait également impliqué dans les tumeurs sporadiques comme semblent l'indiquer les remaniements chromosomiques mis en évidence par caryotype, les quatre ou cinq cas de tumeurs avec délétions somatiques homozygotes, ainsi que quelques cas, rares, de pertes d'allèles limitées à la région 11 p13 [1]

En mars 1989, une trentaine de chercheurs représentant les principaux laboratoires participant à la recherche des gènes situés en $11 \mathrm{p} 13$ et en $11 \mathrm{p} 15$ et à l'étude des différentes formes de tumeurs se réunissaient à
Bethesda pour le premier workshop international consacré au chromosome 11. Cette réunion a permis de dresser l'inventaire des matériels acquis ou réalisés par chaque laboratoire puis de comparer et d'ajuster les différentes cartes physiques obtenues. Bien que des clones correspondant au gène de l'aniridie (AN2) aient été isolés et caractérisés par au moins deux groupes [2, 3], tous les efforts se tournaient vers la région WT en 11p13. Grâce au clone S1 isolé par W. Lewis [4] et localisé dans la région cible, il était clair, au vu de la carte quasiment complète, que le gène devait se rendre dans les mois à venir. Certains avaient déjà un bon candidat, d'autres se montraient beaucoup plus discrets... En dépit de quelques réticences, les deux articles parus récemment l'un dans Nature l'autre dans Cell semblent vouloir donner raison à ces prédictions $[5,6]$.

Cartographie physique ultrafine de la région WAGR. Pendant longtemps les seuls marqueurs disponibles pour étudier la région WAGR ont été le gène de la catalase (CAT) (1980), puis celui de la subunité $\beta$ de l'hormone folliculo-stimulante (FSHB) (1986) et enfin le gène codant pour un antigène de surface MIC1. L'analyse de délétions visibles cytogénétiquement chez des sujets présentant des formes cliniques complètes ou incomplètes du syndrome WAGR, ainsi que l'analyse d'hybrides somatiques établis à partir de deux sujets aniridiques porteurs d'une translocation en $11 \mathrm{p} 13$, apparemment équilibrée, ont été le point de départ d'une cartographie exhaustive de cette région. A partir de 1987, la région ainsi jalonnée par quelques gènes et marqueurs, dans l'ordre suivant : [centromère-CATMIC1-(GU, WT)-AN2-FSHB-téen évidence de pertes d'allèles, à 
lomère], allait faire l'objet d'un effort intense de cartographie physique systématique par des approches parallèles menées de front par une dizaine de laboratoires [7]: (a) la constitution de panels d'hybrides somatiques à partir de sujets porteurs de délétions (54 cas) ou de translocations ( 5 cas) et subdivisant la région en multiples sous-régions ; (b) le clonage de séquences anonymes (une centaine environ pour cette seule région) qui allaient être localisées finement à l'aide de ces hybrides somatiques ou bien par la technique d'électrophorèse en champ pulsé (PFGE) ; (c) l'électrophorèse en champ pulsé, dont la réalisation devenait possible du fait du nombre croissant de marqueurs, permettait d'identifier et d'ordonner chaque fragment de restriction engendré par l'enzyme NotI, puis par d'autres enzymes. La première carte à grande échelle de la région réalisée par le groupe de G. Saunders (Houston) était publiée fin 1988 [8]. En fait, six équipes différentes se consacraient à cette tâche; (d) la digestion de l'ADN, à l'aide d'enzymes de restriction reconnaissant des sites rares riches en dinucléotide $\mathrm{CpG}$, permettait de signaler la présence d'îlots HTF souvent situés dans les régions promotrices de gènes ; (e) la localisation des marqueurs à l'aide d'hybrides somatiques indiquait, en cas d'hybridation croisée avec le parent rongeur, la position de séquences conservées au cours de l'évolution, comme cela est souvent le cas pour les séquences codantes.

A l'inverse de la démarche utilisée pour la recherche du gène de la myopathie de Duchenne et du gène de la mucoviscidose, la cartographie génétique a été pratiquement absente de cette course au gène. La diversité et la multiplicité des remaniements chromosomiques en 11p13 (un minimum de 113 points de cassure différents) ont en effet apporté une subdivision d'une telle finesse qu'elle a permis de dispenser les chercheurs de fastidieuses et certes moins performantes études familiales. Bien que quelques marqueurs polymorphes aient été isolés pour $11 \mathrm{p} 13$, cette région est longtemps restée beaucoup plus pauvre que la région $11 \mathrm{p} 15$. $\mathrm{m} / \mathrm{s} n^{\circ} 5$ vol. 6 , mai 90
Depuis longtemps, en effet, la recherche d'une liaison génétique avec la région $11 \mathrm{p} 13$ restait infructueuse dans les quelques familles dont on disposait, du fait du manque d'informativité des marqueurs. Il aura fallu attendre 1988 pour exclure les deux loci du bras court du 11 dans les cas familiaux!

Deux remaniements chromosomiques ont joué un rôle déterminant dans cette chasse au gène $W T$ : le premier, une délétion constitutionnelle 11 p13 chez un sujet (DAR) non aniridique présentant une tumeur et des anomalies génito-urinaires. Ce remaniement, épargnant le locus de l'aniridie (AN2), a permis de délimiter une frontière entre ce locus et le locus WT [9]. Le second, une délétion homozygote dans une tumeur sporadique (Wit13) révélée par l'absence du marqueur S1. Cette délétion homozygote était en fait la partie délétée commune à des délétions successives chevauchantes sur les deux chromosomes $11[4]$. Des hybrides somatiques ont été réalisés à partir de ces remaniements constitutionnels et somatiques et ont permis de localiser d'autres séquences et surtout d'ordonner les fragments de restriction proches du gène $W T$ et du gène $A N 2$ sur la carte obtenue par électrophorèse en champ pulsé. Cette carte ininterrompue s'étend sur 16 mégabases (soit 0,5\% du génome, soit encore dix fois plus que la région ainsi balayée, pour isoler le gène de la mucoviscidose !). Passée au peigne fin, cette région devait révéler plusieurs zones d'homologie avec d'autres espèces ainsi que la présence de 14 îlots HTF (figure 1).

La marche vers le gène. Pour isoler le gène $W T$, le groupe de G. Bruns (Children's hospital, Boston) [5] a utilisé simultanément les techniques de marche sur le chromosome et des banques de saut. Le point de départ a été une séquence anonyme, 282, localisée à proximité de la région WT/GU et de deux îlots CpG. Repérant par marche sur le chromosome un fragment EcoRI adjacent contenant un site BssHII, une banque de saut BssHII-EcoRI a été utilisée pour un premier saut bidirectionnel. Les deux clones de saut obtenus, effectivement position- nés de part et d'autre de la séquence de départ, ont à leur tour été les points de départ de nouveaux sauts en direction télomérique et centromérique, à l'aide d'une nouvelle banque de saut BssHII/BamHI-HindIII. Les deux clones de saut en direction télomérique ont ensuite permis d'isoler des clones chevauchants à partir de banques de cosmides et de phages et de couvrir environ $60 \mathrm{~kb}$ pour la région la plus télomérique. Ces quatre sauts ont ainsi permis de cloner quatre îlots HTF. Pour deux d'entre eux, les plus télomériques, une hybridation croisée avec l'ADN de rongeur indiquait la présence de séquences conservées. De plus, l'analyse par Northern blot d'ARN fotaux humains a permis de détecter, pour l'un, un transcrit présent dans tous les tissus et, pour l'autre, un transcrit uniquement détectable dans le rein fotal. Le clone génomique correspondant à ce dernier transcrit a ensuite permis d'isoler un $\mathrm{ADNc}$ de $3 \mathrm{~kb}, \mathrm{LK} 15$, à partir d'une banque $\mathrm{ADNc}$ de rein fotal. Le locus génomique correspondant s'étend sur environ $60 \mathrm{~kb}$. Le séquençage complet de ce clone de 3020 nucléotides révèle la présence d'une phase de lecture ouverte sur 1275 nucléotides s'étendant vraisemblablement plus en 5' du fait de l'absence de la séquence consensus des codons d'initiation. Cette séquence comprend deux régions caractéristiques : d'une part, quatre motifs répétés en tandem montrant $63,1 \%$ d'homologie avec la séquence polypeptidique en doigts de zinc du gène EGR2 (early growth response gene, activé durant la transition G0-G1, homologue du gène murin Krox-20) ; et, d'autre part, deux régions riches en proline $(33 \%)$ comparables à celles de la protéine codée par Krox-20 et d'autres protéines se fixant à l'ADN comme le produit du gène Krüppel. Ces régions pourraient représenter des domaines d'activation transcriptionnelle. La recherche de remaniements génomiques par analyse de $65 \mathrm{ADN}$ provenant de tumeurs n'a permis de détecter que deux tumeurs avec délétion homozygote de cette région. La région délétée commune à ces deux tumeurs couvre une séquence de $170 \mathrm{~kb}$, comprise dans le fragment NotI de 
$375 \mathrm{~kb}$ (figure 1) et incluant tous les fragments génomiques $(60 \mathrm{~kb})$ détectés par l'ADNc LK15. Enfin la recherche dans 12 tumeurs analysées par Northern blot de transcrits anormaux, en taille ou en abondance, s'est révélée infructueuse (figure 1).

L'approche utilisée par le groupe de D. Housman du Center For Cancer Research (MIT, Cambridge, USA), en collaboration avec des chercheurs de Toronto (W. Lewis, H. Yeger), est quelque peu différente mais a abouti à l'isolement d'un $\mathrm{ADNc}$ identique [6]. L'un de ces articles [10] décrit la carte complète de la région par PFGE appliquée à une série d'hybrides contenant différentes portions chevauchantes de $11 \mathrm{p} 13$, obtenus par irradiation selon la technique de Goss et Harris d'un hybride somatique (J1-11) ne contenant que le bras court du 11 (C. Jones, Denver, USA). L'inventaire des fragments de restriction a été réalisé par hybridation avec deux séquences humaines correspondant à des séquences répétées : tout d'abord une séquence Alu (représentée environ tous les $10 \mathrm{~kb}$ ) et, ensuite, une séquence correspondant à la famille KpnI. L'ordonnancement des fragments a été réalisé à l'aide de 56 sondes anonymes préalablement localisées et ordonnées). Il a ainsi été reconnu que la position du gène WT était limitée en position distale par le point de cassure de l'hybride DAR et se confondait avec une partie de la région délimitée par la délétion homozygote de la tumeur Wit 13. Cette délétion comprise entre 142 et $345 \mathrm{~kb}$ couvre trois fragments SfiI qui chevauchent les fragments NotI de 500 et $375 \mathrm{~kb}$. L'isolement de l'ADNc décrit dans le deuxième article [6] est essentiellement fondé sur le repérage de deux cosmides situés dans la région $\mathrm{WT}$ reconnue par PFGE. Ces deux clones ont été sélectionnés à partir de 119 clones d'une banque de cosmides établie à l'aide de l'hybride J1-11, sur un panel d'hybrides provenant de patients. L'un de ces clones, J8-3p4 - , montrant une hybridation croisée avec l'ADN de rongeurs et détectant un transcrit parmi des ARN de rein et de rate de Babouin -, a été utilisé

Figure 1. Carte de la région WAGR et marche vers le gène WT. La région $D$ WAGR, encadrée par les gènes CAT et FSHB, comprend, outre les composantes de ce syndrome, le gène de la tumeur de Wilms et des anomalies génitourinaires (WT/GU), le gène de l'aniridie (AN2), le point de cassure d'une translocation dans un syndrome de Potter (GUD) ; la région TTR correspondant aux points de cassure de translocations observées dans des leucémies à cellules $T$. Grâce au clonage de séquences couvrant l'ensemble de cette région et les régions $11 p 12$ et $11 p 14$ adjacentes, la présence de séquences conservées au cours de l'évolution et de séquences riches en CG a pu être repérée. La carte à grande échelle établie par électrophorèse en champs pulsés (PFGE) couvre environ 16 mégabases (Mpb) et a été réalisée par digestion avec l'enzyme Notl puis avec d'autres enzymes, permettant ainsi d'ordonner les clones isolés. Un de ces clones, 282, situé à proximité de deux îlots HTF dans la région WT/GU, dans le fragment Notl de $500 \mathrm{~kb}$, a servi de point de départ pour effectuer un premier saut bidirectionnel $\left(1,1^{\prime}\right)$ à l'aide $d^{\prime}$ une banque de saut. Les séquences ainsi isolées ont à leur tour été utilisées pour un deuxième saut dans les deux directions opposées $\left(2,2^{\prime}\right)$, permettant l'isolement de quatre ilots HTF (emplacements indiqués sur les différents clones par les sites de coupure Bss IIII et Notl: Bs, Nt). Les rectangles gris représentent les sondes (282) qui ont servi de point de départ pour les sauts, rectangles rouges correspondent aux séquences conservées. Les nouveaux clones isolés grâce aux sauts 1 et 2 détectant des séquences conservées ont été utilisés pour rechercher l'expression de transcrits en Northern blots. Un transcrit de $3 \mathrm{~kb}$ détecté avec le clone du saut 2 présentait certaines des propriétés attendues pour le gène WT. Le criblage d'une banque $A D N c$ de rein fœetal a permis d'isoler un $A D N c$ de $3 \mathrm{~kb}$, $L K 15$. La région $5^{\prime}$ de cet $A D N c$ correspond à des séquences génomiques débutant au niveau du site Notl séparant les fragments de $500 \mathrm{~kb}$ et de $375 \mathrm{~kb}$ (d'après Gessler et al., 1990). L'approche utilisée par le groupe de D. Housman qui a abouti à l'isolement de la même séquence est décrite dans le texte.

d'ADNc, de rein embryonnaire, de rein adulte et d'une lignée pré-B. Le transcrit le plus long qui ait été isolé, WT33, est identique, bien que plus court en 5' de 700 bases, à celui trouvé par le groupe de G. Bruns. Il comprend 2313 bases avec une phase de lecture ouverte de 1035 bases qui contient les quatre motifs en doigts de zinc, l'une des deux séquences riches en proline ainsi qu'une séquence riche en sérine et thréonine comparable à celles observées pour des facteurs de transcription comme Sp1. L'analyse de clones génomiques a permis de déterminer l'orientation de l'unité transcriptionnelle correspondante. Celle-ci est orientée dans la direction centromère $\rightarrow$ télomère avec la région 5' comprenant le site NotI séparant les fragments de $500 \mathrm{~kb}$ et de $375 \mathrm{~kb}$. Ce site NotI, inclus dans un îlot $\mathrm{HTF}$, indique vraisemblablement la position de la région promotrice. L'analyse par Northern blot de différents tissus adultes et fœtaux de souris et de babouin révèle un profil d'expression identique à celui observé par l'autre groupe. Cet ADNc détecte un transcrit normal en taille et en abondance dans les WT sporadiques et dans des lignées hémato- poïétiques comme la lignée d'érythroleucémie K562 et la lignée CEM provenant d'une leucémie lymphocytaire aiguë, en accord avec l'expression dans la rate. En revanche, aucun transcrit n'est détecté dans plusieurs autres types de tumeurs (figure 1).

Vrai ou faux candidat? Bien que le groupe de G. Bruns émette quelque réticence à proclamer le gène $W T$ cloné, et cela à juste titre si l'on se souvient des déboires éprouvés par d'autres dans des circonstances analogues, il est tout de même tentant de penser que les arguments en faveur l'emportent sur les réserves. Quelques point demeurent pourtant troublants : la région couverte par les délétions homozygotes, $170 \mathrm{~kb}$, laisse, à côté de ce gène candidat d'environ $60 \mathrm{~kb}$, la place pour d'autres gènes ; la rareté des remaniements génomiques somatiques - deux tumeurs sur 65 - ne reflète pas la fréquence des remaniements germinaux; l'absence de transcrit anormal en taille ou en abondance dans les WT analysées. Les deux derniers points contrastent effectivement avec ce que l'on sait du gène $R B$ - pour lequel des remaniements génomiques et/ou des transcrits anormaux voire absents ont été 


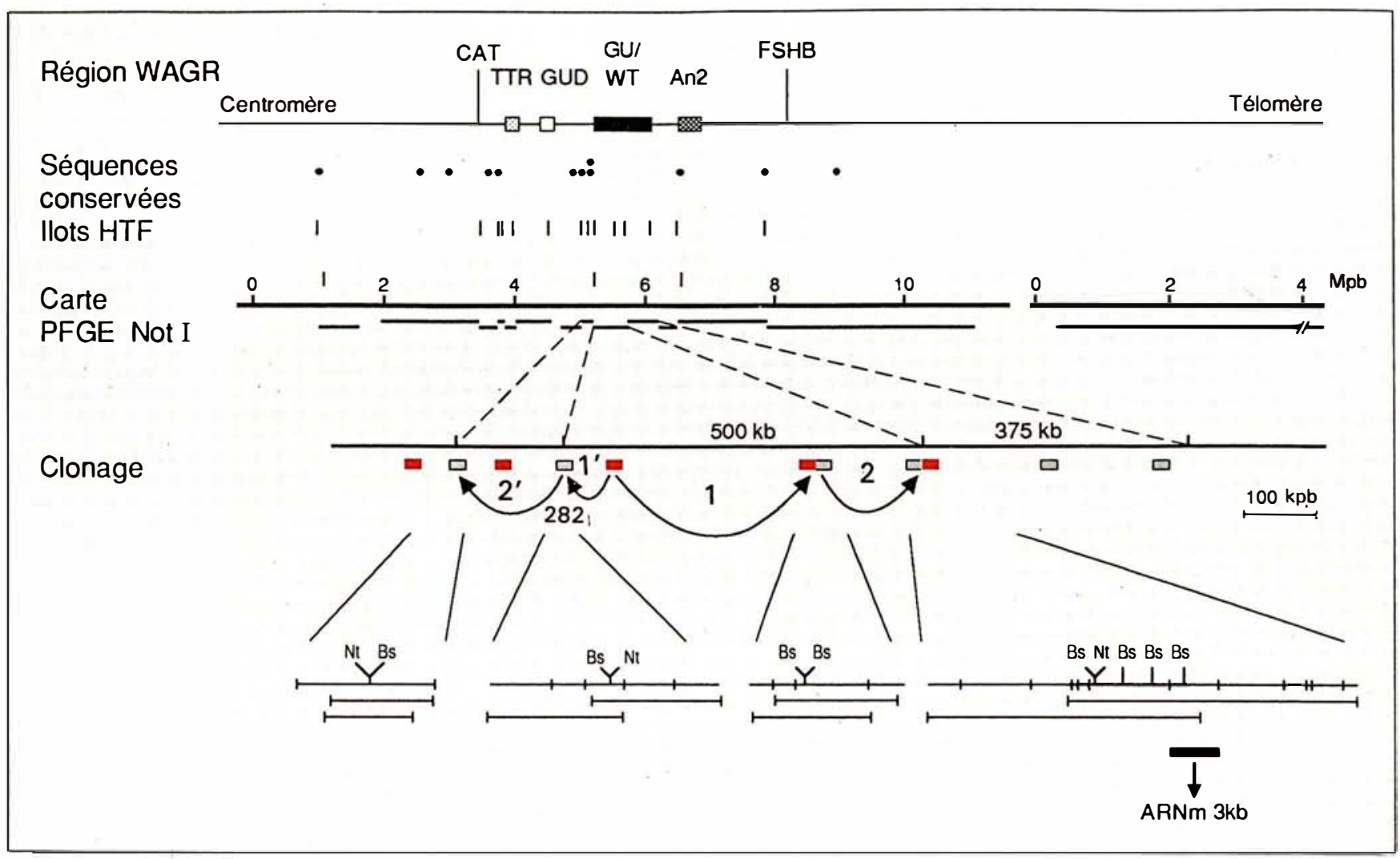

fréquemment observés dans les rétinoblastomes. Ces différences ne sont pas les seules.

- L'expression du gène $R B$ est ubiquitaire et des mutations ont été retrouvées dans des tumeurs très diverses. Le gène candidat $W T$ montre une expression spécifique de tissu restreinte au rein, aux gonades, aux tissus hématopoïétiques et au cœur. Si la fréquence d'autres types de cancer semble effectivement augmentée dans les familles à rétinoblastome, aucune association particulière avec la WT n'a jusqu'à présent été notée. Toutefois, la rareté des formes familiales de WT, l'hétérogénéité génétique et leur pronostic limitent la possibilité d'étudier les cancers secondaires.

- Alors que la protéine RB se fixe in vitro à l'ADN, il ne semble pas que cette fixation se fasse de manière spécifique, du fait de l'absence de séquences caractéristiques. La protéine p105RB semble avoir pour fonction principale de contrôler la croissance cellulaire par l'intermé- diaire d'interactions avec d'autres protéines et surtout par le biais d'étapes de phosphorylations/déphosphorylations. Le produit du gène $W T$, qui ne montre pas d'homologie avec le produit du gène $R B$, possède en revanche des caractéristiques flagrantes d'une DNA binding protein et surtout d'un facteur de transcription.

- Il n'existe qu'un seul gène de prédisposition pour le rétinoblastome alors qu'il y a au moins trois loci distincts de prédisposition à la $W T$. La mise en évidence de pertes d'allèles pour la région $11 \mathrm{p} 15$ dans les tumeurs de sujets avec syndrome WAGR et dans des cas familiaux (avec altération du troisième locus) montre qu'une tumeur peut présenter simultanément des altérations à ces différents loci. Sont-elles nécessaires ? Doivent-elles se produire dans un ordre déterminé ? Existe-t-il des interactions entre ces loci et leurs produits ? Peut-il exister des diagrammes différents d'altérations définissant des sous-groupes de tumeurs? Il faudra attendre probablement l'identification du produit du gène $\mathrm{WT}$ et le clonage des deux autres gènes pour obtenir un début de réponse à ces questions. En dépit de ces différences inattendues avec le gène $R b$, le gène $W T$ candidat réunit un certain nombre de propriétés compatibles avec son rôle dans le développement de la WT. 1. Son expression au cours du développement rénal, de la $7 \mathrm{e}$ à la $18^{\mathrm{e}}$ semaine, aussi bien dans le mésonéphros que dans le métanéphros, rappelle la persistance de cellules peu différenciées de type blastème, voire d'une néphroblastomatose dans les reins d'enfants développant des $W T$, et surtout l'aspect peu différencié, dit embryonnaire, des tumeurs ellesmêmes (V. van Heyningen, communication personnelle);

2. Son expression dans les gonades, chez l'embryon et au cours de la vie fotale, est compatible avec l'hypothèse selon laquelle le gène $W T$ peut avoir des effets pléiotropes à la fois sur le développement rénal et génital (V. van Heyningen, communication personnelle). Cela pourrait d'ail- 


\section{${ }^{*}$ GLOSSAIRE*}

Banque de saut : technique qui consiste $\dot{a}$ couper de grands fragments d'ADN (autour de $100 \mathrm{kpb}$ ), à les recirculariser en liant leurs deux extrémités à un marqueur de sélection, puis à cloner, après une nouvelle coupure avec une autre enzyme, l'ADN des deux extrémités maintenant réunies au marqueur. Si un tel clone est détecté par hybridation avec une sonde de l'une des extrémités, l'ADN de l'autre extrémité fournira lui-même une sonde correspondant à une région du chromosome située, dans l'exemple pris ici, $100 \mathrm{kpb}$ plus loin.

Cryptorchidie : absence de migration des testicules dans les bourses.

Hypospadias : malformation congénitale de l'urètre de l'homme, caractérisée par un orifice anormal situé à une distance variable de l'extrémité du gland.

Ilots HTF (Hpall tiny fragments) ilots contenant de nombreux sites de coupure par l'enzyme de restriction Hpall, qui reconnaît la séquence 5' CCGG 3' lorsque les deux $C$ sont déméthylés. Des régions riches en $C G$ déméthylées sont caractéristiques des séquences bordantes 5' des gènes actifs, principalement ceux à expression ubiquitaire, mais également de certains gènes à expression tissu-spécifique.

Technique de Goss et Harris : un hybride somatique contenant le chromosome $11(J 1-11)$ est soumis à une irradiation de 4000 à 8000 rad qui fractionne les chromosomes. Ces cellules irradiées sont ensuite fusionnées avec des cellules de hamster. Les hybrides ainsi obtenus par fusion et qui ont retenu la région 11 p13 sont sélectionnés à l'aide d'un anticorps reconnaissant l'antigène MIC1 localisé en 11p13. Une fraction plus ou moins grande de la région 11 p13 peut ainsi être intégrée dans les chromosomes de hamster. Cette technique peut être utilisée pour la construction de panels d'hybrides somatiques spécifiques d'autres régions chromosomiques. leurs rendre compte de la fréquence accrue de cryptorchidie et d'hypospadias chez les enfants de sexe mâle avec $W T$, qu'elle soit ou non associée à une aniridie. Il reste pourtant un point non éclairci : le point de cassure d'une translocation chez un enfant avec syndrome de Potter (agénésie rénale) se trouve en position beaucoup plus centromérique que le gène candidat $W T$. Mais la région WAGR contient plusieurs autres îlots HTF et peut-être autant de gènes... 3. L'absence de remaniements génomiques détectables ou de messagers anormaux pourrait s'expliquer par l'existence de hot-spots, de mutations touchant des régions clés comme les doigts de zinc ou les régions riches en proline. Mutations qui, à l'instar de celles observées pour un autre anti-oncogène, la p53, pourraient être préférentiellement des mutations ponctuelles et/ou avoir un caractère dominant. Enfin il n'est pas impossible que les délétions de la région $11 \mathrm{p} 13$ favorisent, par un retard de différenciation, un asynchronisme de différents types cellulaires responsable de l'émission de signaux inappropriés.

L'existence d'un modèle animal serait à cet égard particulièrement précieux : la technologie des animaux transgéniques devrait être applicable à condition que le diagramme de différenciation soit similaire? Le groupe de D. Housman a identifié une souris porteuse d'une délétion du chromosome 2 correspondant à la région WAGR. Cette souris présente une anomalie du développement de l'œil (small eye) qui pourrait correspondre à la délétion du locus AN2. Pourtant, bien que la délétion englobe la région du gène candidat $W T$ (LK15), cet animal ne développe pas de tumeur. Ces différences de pathogénicité d'une même délétion entre les deux espèces rappelle une situation analogue pour la myopathie de Duchenne. Elle n'est cependant pas surprenante quand on connaît les différences du développement rénal au cours de la vie fotale entre les deux espèces. Des pertes d'allèles pour la région homologue de la région $11 \mathrm{p} 15$ (chromosome 7 de la souris) ont également été observées dans des carcinomes chimio-induits de la souris. On pour- rait ainsi envisager d'induire des tumeurs chez les animaux génétiquement prédisposés pour étudier le rôle respectif de chacun des deux loci. Enfin l'expression du gène $W T$ dans les lignées hématopoïétiques pourrait représenter un modèle d'étude plus maniable. En cas d'altération du gène $W T$, les lignées d'hémopathies malignes et les cellules hématopoïétiques devraient se prêter plus facilement à des expériences de restauration du phénotype non transformé par introduction du gène $W T$. Des expériences de ce type ont déjà été réalisées avec succès par transfert d'un chromosome 11 normal dans une lignée de WT. Pourtant trois faits invitent à la prudence quant à l'interprétation de ces résultats ou à leur reproductibilité avec un gène cloné : (a) il ne faut pas oublier que le bras court du 11 possède deux gènes de susceptibilité à la WT ; (b) par des expériences analogues de fusion cellulaire, il a été montré que la région du chromosome 11 faisant perdre à des cellules Hela leur caractère tumorigène était en fait le bras long du chromosome 11!; (c) la perte du caractère tumorigène de lignées de rétinoblastome obtenue par transfection à l'aide d'un rétrovirus porteur du gène $R B$ fait l'objet d'une vive controverse car ces résultats n'ont jamais pu être reproduits par un autre laboratoire.

Si le clonage du gène $R B$ apportait la possibilité d'un diagnostic des individus à risque dans les cas familiaux (30 à $40 \%$ des cas), ou la mise en évidence de mutations survenues de novo (un tiers des cas familiaux) permettant de connaître le risque de récidive et d'adapter la surveillance, l'impact de la découverte de ce gène $W T$ est plus limité pour l'instant en termes de diagnostic. En effet, la proportion de cas de WT relevant d'un conseil génétique est faible (quelques \%) et surtout l'hétérogénéité génétique interdit pratiquement l'utilisation de cette sonde pour un diagnostic avant que l'on connaisse la part exacte prise par chacun des gènes. En revanche, le clonage de ce gène est beaucoup plus qu'une page tournée, c'est une porte qui s'ouvre sur un modèle d'étude des relations entre anomalies du développement et 
prédisposition à des cancers. Il devrait nous permettre de franchir des étapes importantes dans un domaine où les connaissances nous font encore tant défaut: celui des tumeurs de l'enfant

\section{Claudine Junien}

Professeur de génétique à

Paris-Ouest, université René-Descartes.

Inserm U.73, château de Longchamp, Bois de Boulogne, 75016 Paris, France.

\section{RÉFÉRENCES}

1. Junien C, Henry I. Bras court du chromosome 11 : empreinte parentale différentielle, tumorigenèse et pertes d'allèles. médecine/sciences $1989 ; 5: 480-8$.

2. Davis LM, Stallard R, Thomas GH, et al. Two anonymous DNA segments distinguish the Wilms' tumor and aniridia loci within the WAGR complex. Science, 1988 ; $241: 840-2$

3. Gessler M, Simola KOJ, Bruns GAP. Cloning of breakpoints of a chromosome translocation identifies the AN2 locus. Science $1989 ; 244$ : 1575-8.

4. Lewis $\mathrm{WH}$, Yeger $\mathrm{H}$, Bonetta L, et al. Homozygous deletion of a DNA marker chromosome $11 \mathrm{p} 13$ in sporadic Wilms' tumor. Genomics 1988; 3 : 25-31.

5. Gessler M, Poustka A, Cavenee W, Neve RL, Orkin SH, Bruns GAP. Homozygous deletion in Wilms' tumours of a zinc-finger gene identified by chromosome jumping. Nature 1990 ; 343 : 774-8.

6. Call KM, Glaser T, Ito CY, et al. Isolation and characterization of a zinc finger polypeptide gene at the human chromosome 11 Wilms' tumor locus. Cell 1990 ; $60: 509-20$.
7. Junien C, McBride WO. Report of the committee on the genetic constitution of chromosome 11. Cytogenet Cell Genet 1989 ; $51: 226-58$.

8. Compton DA, Weil MM, Jones C, Riccardi VM, Strong LC, Saunders GF. Long range physical map of the Wilms' tumoraniridia region on human chromosome 11 . Cell $1988 ; 55: 827-36$

9. Couillin P. Characterization of a panel of somatic cell hybrids for subregional mapping along $11 \mathrm{p}$ and within band $11 \mathrm{p} 13$. Hum Genet 1989 ; 82 : 171-8.

10. Rose EA, Glaser T, Jones C, et al. Complete physical map of the WAGR region of $11 \mathrm{p} 13$ localizes a candidate Wilms' tumor gene. Cell $1990 ; 60: 495-508$.

11. Lee $\mathrm{WH}$, Bookstein $\mathrm{R}$, Mong $\mathrm{R}$, Young LJ, Shew JY, Lee EMP. Human retinoblastoma susceptibility gene : cloning, identification, and sequence. Science 1987 ; 235 : 1394-9.

12. Friend SM, Morowitz JM, Gerber MR, et al. Deletions of a DNA sequence in retinoblastomas and mesenchymal tumors : organization of the sequence and its encoded protein. Proc Natl Acad Sci USA 1987 ; 84 : 9059-63. 